\title{
A New Hybrid Iterative Algorithm for Fixed-Point Problems, Variational Inequality Problems, and Mixed Equilibrium Problems
}

\author{
Yonghong Yao, ${ }^{1}$ Yeong-Cheng Liou, ${ }^{2}$ and Jen-Chih Yao ${ }^{3}$ \\ ${ }^{1}$ Department of Mathematics, Tianjin Polytechnic University, Tianjin 300160, China \\ ${ }^{2}$ Department of Information Management, Cheng Shiu University, Kaohsiung 833, Taiwan \\ ${ }^{3}$ Department of Applied Mathematics, National Sun Yat-Sen University, Kaohsiung 804, Taiwan
}

Correspondence should be addressed to Jen-Chih Yao, yaojc@math.nsysu.edu.tw

Received 29 August 2007; Accepted 6 February 2008

Recommended by Tomonari Suzuki

\begin{abstract}
We introduce a new hybrid iterative algorithm for finding a common element of the set of fixed points of an infinite family of nonexpansive mappings, the set of solutions of the variational inequality of a monotone mapping, and the set of solutions of a mixed equilibrium problem. This study, proves a strong convergence theorem by the proposed hybrid iterative algorithm which solves fixed-point problems, variational inequality problems, and mixed equilibrium problems.

Copyright $(2008$ Yonghong Yao et al. This is an open access article distributed under the Creative Commons Attribution License, which permits unrestricted use, distribution, and reproduction in any medium, provided the original work is properly cited.
\end{abstract}

\section{Introduction}

Let $C$ be a nonempty closed convex subset of a real Hilbert space $H$. Recall that a mapping $f: C \rightarrow C$ is called contractive if there exists a constant $\alpha \in(0,1)$ such that $\|f(x)-f(y)\| \leq$ $\alpha\|x-y\|$ for all $x, y \in C$. A mapping $T: C \rightarrow C$ is said to be nonexpansive if $\|T x-T y\| \leq\|x-y\|$ for all $x, y \in C$. Denote the set of fixed points of $T$ by $F(T)$.

Let $\varphi: C \rightarrow \mathbf{R}$ be a real-valued function and $\Theta: C \times C \rightarrow \mathbf{R}$ be an equilibrium bifunction, that is, $\Theta(u, u)=0$ for each $u \in C$. The mixed equilibrium problem (for short, MEP) is to find $x^{*} \in C$ such that

$$
\text { MEP: } \Theta\left(x^{*}, y\right)+\varphi(y)-\varphi\left(x^{*}\right) \geq 0 \quad \forall y \in C .
$$

In particular, if $\varphi \equiv 0$, this problem reduces to the equilibrium problem (for short, EP), which is to find $x^{*} \in C$ such that

$$
\text { EP: } \Theta\left(x^{*}, y\right) \geq 0 \quad \forall y \in C \text {. }
$$


Denote the set of solutions of MEP by $\Omega$. The mixed equilibrium problems include fixedpoint problems, optimization problems, variational inequality problems, Nash equilibrium problems, and the equilibrium problems as special cases (see, e.g., [1-5]). Some methods have been proposed to solve the MEP and EP (see, e.g., [5-14]). In 1997, Combettes and Hirstoaga [13] introduced an iterative method of finding the best approximation to the initial data and proved a strong convergence theorem. Subsequently, S. Takahashi and W. Takahashi [8] introduced another iterative scheme for finding a common element of the set of solutions of EP and the set of fixed-point points of a nonexpansive mapping. Yao et al. [12] considered an iterative scheme for finding a common element of the set of solutions of EP and the set of common fixed points of an infinite nonexpansive mappings. Very recently, Zeng and Yao [14] considered a new iterative scheme for finding a common element of the set of solutions of MEP and the set of common fixed points of finitely many nonexpansive mappings. Their results extend and improve many results in the literature.

Let $A$ of $C$ into $H$ be a nonlinear mapping. It is well known that the variational inequality problem is to find $u \in C$ such that

$$
\langle A u, v-u\rangle \geq 0 \quad \forall v \in C .
$$

The set of solutions of the variational inequality problem is denoted by $\operatorname{VI}(C, A)$. A mapping $A: C \rightarrow H$ is called $\beta$-inverse-strongly monotone if there exists a positive real number $\beta$ such that

$$
\langle A u-A v, u-v\rangle \geq \beta\|A u-A v\|^{2} \quad \forall u, v \in C .
$$

Recently, some authors have proposed new iterative algorithms to approximate a common element of the set of fixed points of a nonxpansive mapping and the set of solutions of the variational inequality. For the details, see $[15,16]$ and the references therein.

Motivated by the recent works, in this paper we introduce a new hybrid iterative algorithm for finding a common element of the set of fixed points of an infinite family of nonexpansive mappings, the set of solutions of the variational inequality of a monotone mapping, and the set of solutions of a mixed equilibrium problem. We prove a strong convergence theorem by the proposed hybrid iterative algorithm which solves fixed-point problems, variational inequality problems, and mixed equilibrium problems.

\section{Preliminaries}

Let $H$ be a real Hilbert space with inner product $\langle\cdot, \cdot\rangle$ and norm $\|\cdot\|$. Let $C$ be a nonempty closed convex subset of $H$. Then for any $x \in H$, there exists a unique nearest point in $C$, denoted by $P_{C}(x)$ such that

$$
\left\|x-P_{C}(x)\right\| \leq\|x-y\| \quad \forall y \in C .
$$

Such a $P_{C}$ is called the metric projection of $H$ onto $C$. It is well known that $P_{C}$ is a nonexpansive mapping and satisfies

$$
\left\langle x-y, P_{C} x-P_{C} y\right\rangle \geq\left\|P_{C} x-P_{C} y\right\|^{2} \quad \forall x, y \in H .
$$


Moreover, $P_{C}$ is characterized by the following properties:

$$
\begin{gathered}
\left\langle x-P_{C} x, y-P_{C} x\right\rangle \leq 0 \\
\|x-y\|^{2} \geq\left\|x-P_{C} x\right\|^{2}+\left\|y-P_{C} x\right\|^{2} \quad \forall x \in H, y \in C .
\end{gathered}
$$

It is clear that

$$
u \in V I(C, A) \Longleftrightarrow u=P_{C}(u-\lambda A u) \quad \forall \lambda>0 .
$$

In this paper, for solving the mixed equilibrium problems for an equilibrium bifunction $\Theta: C \times C \rightarrow \mathbf{R}$, we assume that $\Theta$ satisfies the following conditions:

(H1) $\Theta$ is monotone, that is, $\Theta(x, y)+\Theta(y, x) \leq 0$ for all $x, y \in C$;

(H2) for each fixed $y \in C, x \mapsto \Theta(x, y)$ is concave and upper semicontinuous;

(H3) for each $x \in C, y \mapsto \Theta(x, y)$ is convex.

A mapping $\eta: C \times C \rightarrow H$ is called Lipschitz continuous if there exists a constant $\lambda>0$ such that

$$
\|\eta(x, y)\| \leq \lambda\|x-y\| \quad \forall x, y \in C
$$

A differentiable function $K: C \rightarrow \mathbf{R}$ on a convex set $C$ is called:

(i) $\eta$-convex if

$$
K(y)-K(x) \geq\left\langle K^{\prime}(x), \eta(y, x)\right\rangle \quad \forall x, y \in C,
$$

where $K^{\prime}$ is the Fréchet derivative of $K$ at $x$;

(ii) $\eta$-strongly convex if there exists a constant $\sigma>0$ such that

$$
K(y)-K(x)-\left\langle K^{\prime}(x), \eta(y, x)\right\rangle \geq\left(\frac{\sigma}{2}\right)\|x-y\|^{2} \quad \forall x, y \in C
$$

Let $C$ be a nonempty closed convex subset of a real Hilbert space $H, \varphi: C \rightarrow \mathbf{R}$ be a realvalued function, and $\Theta: C \times C \rightarrow \mathbf{R}$ be an equilibrium bifunction. Let $r$ be a positive number. For a given point $x \in C$, the auxiliary problem for MEP consists of finding $y \in C$ such that

$$
\Theta(y, z)+\varphi(z)-\varphi(y)+\frac{1}{r}\left\langle K^{\prime}(y)-K^{\prime}(x), \eta(z, y)\right\rangle \geq 0 \quad \forall z \in C .
$$

Let $S_{r}: C \rightarrow C$ be the mapping such that for each $x \in C, S_{r}(x)$ is the solution set of the auxiliary problem MEP, that is,

$$
S_{r}(x)=\left\{y \in C: \Theta(y, z)+\varphi(z)-\varphi(y)+\frac{1}{r}\left\langle K^{\prime}(y)-K^{\prime}(x), \eta(z, y)\right\rangle \geq 0 \forall z \in C\right\} \quad \forall x \in C .
$$

We first need the following important and interesting result. 
Lemma 2.1 (see [14]). Let $C$ be a nonempty closed convex subset of a real Hilbert space $H$ and let $\varphi: C \rightarrow \mathbf{R}$ be a lower semicontinuous and convex functional. Let $\Theta: C \times C \rightarrow \mathbf{R}$ be an equilibrium bifunction satisfying conditions (H1)-(H3). Assume that

(i) $\eta: C \times C \rightarrow H$ is Lipschitz continuous with constant $\lambda>0$ such that

(a) $\eta(x, y)+\eta(y, x)=0$ for all $x, y \in C$,

(b) $\eta(\cdot, \cdot)$ is affine in the first variable,

(c) for each fixed $y \in C, x \mapsto \eta(y, x)$ is sequentially continuous from the weak topology to the weak topology;

(ii) $K: C \rightarrow \mathbf{R}$ is $\eta$-strongly convex with constant $\sigma>0$ and its derivative $K^{\prime}$ is sequentially continuous from the weak topology to the strong topology;

(iii) for each $x \in C$, there exist a bounded subset $D_{x} \subset C$ and $z_{x} \in C$ such that for any $y \in C \backslash D_{x}$,

$$
\Theta\left(y, z_{x}\right)+\varphi\left(z_{x}\right)-\varphi(y)+\frac{1}{r}\left\langle K^{\prime}(y)-K^{\prime}(x), \eta\left(z_{x}, y\right)\right\rangle<0
$$

Then there hold the following:

(i) $S_{r}$ is single-valued;

(ii) $S_{r}$ is nonexpansive if $K^{\prime}$ is Lipschitz continuous with constant $v>0$ such that $\sigma \geq \lambda v$ and

$$
\left\langle K^{\prime}\left(x_{1}\right)-K^{\prime}\left(x_{2}\right), \eta\left(u_{1}, u_{2}\right)\right\rangle \geq\left\langle K^{\prime}\left(u_{1}\right)-K^{\prime}\left(u_{2}\right), \eta\left(u_{1}, u_{2}\right)\right\rangle \quad \forall\left(x_{1}, x_{2}\right) \in C \times C,
$$

where $u_{i}=S_{r}\left(x_{i}\right)$ for $i=1,2$;

(iii) $F\left(S_{r}\right)=\Omega$;

(vi) $\Omega$ is closed and convex.

We also need the following lemmas for proving our main results.

Lemma 2.2 (see [17]). Let $\left\{x_{n}\right\}$ and $\left\{z_{n}\right\}$ be bounded sequences in a Banach space $X$ and let $\left\{\beta_{n}\right\}$ be a sequence in $[0,1]$ with $0<\liminf _{n \rightarrow \infty} \beta_{n} \leq \limsup _{n \rightarrow \infty} \beta_{n}<1$. Suppose $x_{n+1}=\left(1-\beta_{n}\right) z_{n}+\beta_{n} x_{n}$ for all integers $n \geq 0$ and $\lim \sup _{n \rightarrow \infty}\left(\left\|z_{n+1}-z_{n}\right\|-\left\|x_{n+1}-x_{n}\right\|\right) \leq 0$. Then $\lim _{n \rightarrow \infty}\left\|z_{n}-x_{n}\right\|=0$.

Lemma 2.3 (see [18]). Assume $\left\{a_{n}\right\}$ is a sequence of nonnegative real numbers such that $a_{n+1} \leq$ $\left(1-\gamma_{n}\right) a_{n}+\delta_{n}$, where $\left\{\gamma_{n}\right\}$ is a sequence in $(0,1)$ and $\left\{\delta_{n}\right\}$ is a sequence such that

(1) $\sum_{n=1}^{\infty} \gamma_{n}=\infty$;

(2) $\lim \sup _{n \rightarrow \infty} \delta_{n} / \gamma_{n} \leq 0$ or $\sum_{n=1}^{\infty}\left|\delta_{n}\right|<\infty$.

Then $\lim _{n \rightarrow \infty} a_{n}=0$.

\section{Iterative algorithm and strong convergence theorems}

In this section, we first introduce a new iterative algorithm. Consequently, we will establish a strong convergence theorem for this iteration algorithm. To be more specific, let $T_{1}, T_{2}, \ldots$ be 
infinite mappings of $C$ into itself and let $\xi_{1}, \xi_{2}, \ldots$ be real numbers such that $0 \leq \xi_{i} \leq 1$ for every $i \in \mathbf{N}$. For any $n \in \mathbf{N}$, define a mapping $W_{n}$ of $C$ into itself as follows:

$$
\begin{gathered}
U_{n, n+1}=I, \\
U_{n, n}=\xi_{n} T_{n} U_{n, n+1}+\left(1-\xi_{n}\right) I, \\
U_{n, n-1}=\xi_{n-1} T_{n-1} U_{n, n}+\left(1-\xi_{n-1}\right) I, \\
\vdots \\
U_{n, k}=\xi_{k} T_{k} U_{n, k+1}+\left(1-\xi_{k}\right) I, \\
U_{n, k-1}=\xi_{k-1} T_{k-1} U_{n, k}+\left(1-\xi_{k-1}\right) I, \\
\vdots \\
U_{n, 2}=\xi_{2} T_{2} U_{n, 3}+\left(1-\xi_{2}\right) I, \\
W_{n}=U_{n, 1}=\xi_{1} T_{1} U_{n, 2}+\left(1-\xi_{1}\right) I .
\end{gathered}
$$

Such $W_{n}$ is called the $W$-mapping generated by $T_{n}, T_{n-1}, \ldots, T_{2}, T_{1}$ and $\xi_{n}, \xi_{n-1}, \ldots, \xi_{2}, \xi_{1}$. For the iterative algorithm for a finite family of nonexpansive mappings, we refer the reader to [19].

We have the following crucial Lemmas 3.1 and 3.2 concerning $W_{n}$ which can be found in [20]. Now we only need the following similar version in Hilbert spaces.

Lemma 3.1. Let $C$ be a nonempty closed convex subset of a real Hilbert space $H$. Let $T_{1}, T_{2}, \ldots$ be nonexpansive mappings of $C$ into itself such that $\bigcap_{n=1}^{\infty} F\left(T_{n}\right)$ is nonempty, and let $\xi_{1}, \xi_{2}, \ldots$ be real numbers such that $0<\xi_{i} \leq b<1$ for any $i \in \mathbf{N}$. Then for every $x \in C$ and $k \in \mathbf{N}$, the limit $\lim _{n \rightarrow \infty} U_{n, k} x$ exists.

Lemma 3.2. Let $C$ be a nonempty closed convex subset of a real Hilbert space $H$. Let $T_{1}, T_{2}, \ldots$ be nonexpansive mappings of $C$ into itself such that $\bigcap_{n=1}^{\infty} F\left(T_{n}\right)$ is nonempty, and let $\xi_{1}, \xi_{2}, \ldots$ be real numbers such that $0<\xi_{i} \leq b<1$ for any $i \in N$. Then $F(W)=\bigcap_{n=1}^{\infty} F\left(T_{n}\right)$.

The following remark [12] is important to prove our main results.

Remark 3.3. Using Lemma 3.1, one can define a mapping $W$ of $C$ into itself as $W x=$ $\lim _{n \rightarrow \infty} W_{n} x=\lim _{n \rightarrow \infty} U_{n, 1} x$ for every $x \in C$. If $\left\{x_{n}\right\}$ is a bounded sequence in $C$, then we have

$$
\lim _{n \rightarrow \infty}\left\|W x_{n}-W_{n} x_{n}\right\|=0
$$

Throughout this paper, we will assume that $0<\xi_{i} \leq b<1$ for every $i \in \mathbf{N}$.

Now we introduce the following iteration algorithm.

Algorithm 3.4. Let $r>0$ be a constant. Let $\varphi: C \rightarrow \mathbf{R}$ be a lower semicontinuous and convex functional and let $\Theta: C \times C \rightarrow \mathbf{R}$ be an equilibrium bifunction. Let $A: C \rightarrow H$ be a $\beta$ inverse-strongly monotone mapping and $W_{n}$ be the $W$-mapping defined by (3.1). Let $f$ be a 
contraction of $C$ into itself with coefficient $\alpha \in(0,1)$ and given $x_{0} \in C$ arbitrarily. Suppose that the sequences $\left\{x_{n}\right\}$ and $\left\{y_{n}\right\}$ are generated iteratively by

$$
\begin{gathered}
\Theta\left(z_{n}, x\right)+\varphi(x)-\varphi\left(z_{n}\right)+\frac{1}{r}\left\langle K^{\prime}\left(z_{n}\right)-K^{\prime}\left(x_{n}\right), \eta\left(x, z_{n}\right)\right\rangle \geq 0 \quad \forall x \in C, \\
y_{n}=P_{C}\left(z_{n}-\lambda_{n} A z_{n}\right) \\
x_{n+1}=\alpha_{n} f\left(W_{n} x_{n}\right)+\beta_{n} x_{n}+\gamma_{n} W_{n} P_{C}\left(y_{n}-\lambda_{n} A y_{n}\right) \quad \forall n \geq 0
\end{gathered}
$$

where $\left\{\alpha_{n}\right\},\left\{\beta_{n}\right\}$, and $\left\{\gamma_{n}\right\}$ are three sequences in $(0,1)$, and $\left\{\lambda_{n}\right\}$ is a sequence in $[0,2 \beta]$.

Now we study the strong convergence of the hybrid iterative algorithm (3.3).

Theorem 3.5. Let $C$ be a nonempty closed convex subset of a real Hilbert space $H$ and let $\varphi: C \rightarrow \mathbf{R}$ be a lower semicontinuous and convex functional. Let $\Theta: C \times C \rightarrow \mathbf{R}$ be an equilibrium bifunction satisfying conditions (H1)-(H3) and let $T_{1}, T_{2}, \ldots$ be an infinite family of nonexpansive mappings of $C$ into itself. Let $A: C \rightarrow H$ be a $\beta$-inverse-strongly monotone mapping such that $\cap_{n=1}^{\infty} F\left(T_{n}\right) \cap V I(A, C) \cap$ $\Omega \neq \varnothing$. Suppose $\left\{\alpha_{n}\right\},\left\{\beta_{n}\right\}$, and $\left\{\gamma_{n}\right\}$ are three sequences in $(0,1)$ with $\alpha_{n}+\beta_{n}+\gamma_{n}=1, n \geq 0$. Assume that

(i) $\eta: C \times C \rightarrow H$ is Lipschitz continuous with constant $\lambda>0$ such that

(a) $\eta(x, y)+\eta(y, x)=0$ for all $x, y \in C$,

(b) $\eta(\cdot, \cdot)$ is affine in the first variable,

(c) for each fixed $y \in C, x \mapsto \eta(y, x)$ is sequentially continuous from the weak topology to the weak topology;

(ii) $K: C \rightarrow \mathbf{R}$ is $\eta$-strongly convex with constant $\sigma>0$ and its derivative $K^{\prime}$ is not only sequentially continuous from the weak topology to the strong topology but also Lipschitz continuous with constant $v>0$ such that $\sigma \geq \lambda \mathcal{\nu}$;

(iii) for each $x \in C$; there exist a bounded subset $D_{x} \subset C$ and $z_{x} \in C$ such that for any $y \in C \backslash D_{x}$,

$$
\Theta\left(y, z_{x}\right)+\varphi\left(z_{x}\right)-\varphi(y)+\frac{1}{r}\left\langle K^{\prime}(y)-K^{\prime}(x), \eta\left(z_{x}, y\right)\right\rangle<0
$$

(iv) $\lim _{n \rightarrow \infty} \alpha_{n}=0, \sum_{n=0}^{\infty} \alpha_{n}=\infty, 0<\liminf _{n \rightarrow \infty} \beta_{n} \leq \limsup _{n \rightarrow \infty} \beta_{n}<1, \lambda_{n} \in[a, b] \subset$ $(0,2 \beta)$, and $\lim _{n \rightarrow \infty}\left(\lambda_{n+1}-\lambda_{n}\right)=0$.

Let $f$ be a contraction of $C$ into itself and given $x_{0} \in C$ arbitrarily. Then the sequence $\left\{x_{n}\right\}$ generated by (3.3) converges strongly to $x^{*}=P_{\Gamma} f\left(x^{*}\right)$, where $\Gamma=\cap_{n=1}^{\infty} F\left(T_{n}\right) \cap V I(A, C) \cap \Omega$ provided that $S_{r}$ is firmly nonexpansive.

Proof. We first note that $f$ is a contraction with coefficient $\alpha \in(0,1)$. Then $\left\|P_{\Gamma} f(x)-P_{\Gamma} f(y)\right\| \leq$ $\|f(x)-f(y)\| \leq \alpha\|x-y\|$ for all $x, y \in C$. Therefore $P_{\Gamma} f$ is a contraction of $C$ into itself which implies that there exists a unique element $x^{*} \in C$ such that $x^{*}=P_{\Gamma} f\left(x^{*}\right)$.

Next we divide the following proofs into several steps.

Step $1\left(\left\{x_{n}\right\},\left\{y_{n}\right\}\right.$, and $\left\{z_{n}\right\}$ are bounded). Let $x^{*} \in \Gamma$. From the definition of $S_{r}$, we know that $z_{n}=S_{r} x_{n}$. It follows that

$$
\left\|z_{n}-x^{*}\right\|=\left\|S_{r} x_{n}-S_{r} x^{*}\right\| \leq\left\|x_{n}-x^{*}\right\| .
$$


Yonghong Yao et al.

For all $x, y \in C$ and $\lambda_{n} \in[0,2 \beta]$, we note that

$$
\begin{aligned}
\left\|\left(I-\lambda_{n} A\right) x-\left(I-\lambda_{n} A\right) y\right\|^{2} & =\left\|(x-y)-\lambda_{n}(A x-A y)\right\|^{2} \\
& =\|x-y\|^{2}-2 \lambda_{n}\langle A x-A y, x-y\rangle+\lambda_{n}^{2}\|A x-A y\|^{2} \\
& \leq\|x-y\|^{2}+\lambda_{n}\left(\lambda_{n}-2 \beta\right)\|A x-A y\|^{2},
\end{aligned}
$$

which implies that $I-\lambda_{n} A$ is nonexpansive.

Set $u_{n}=P_{C}\left(y_{n}-\lambda_{n} A y_{n}\right)$ for all $n \geq 0$. From (2.4), we have that $x^{*}=P_{C}\left(x^{*}-\lambda_{n} A x^{*}\right)$. It follows from (3.6) that

$$
\begin{aligned}
\left\|y_{n}-x^{*}\right\| & =\left\|P_{C}\left(z_{n}-\lambda_{n} A z_{n}\right)-P_{C}\left(x^{*}-\lambda_{n} A x^{*}\right)\right\| \\
& \leq\left\|\left(z_{n}-\lambda_{n} A z_{n}\right)-\left(x^{*}-\lambda_{n} A x^{*}\right)\right\| \leq\left\|z_{n}-x^{*}\right\| \leq\left\|x_{n}-x^{*}\right\|, \\
\left\|u_{n}-x^{*}\right\| & =\left\|P_{C}\left(y_{n}-\lambda_{n} A y_{n}\right)-P_{C}\left(x^{*}-\lambda_{n} A x^{*}\right)\right\| \\
& \leq\left\|\left(y_{n}-\lambda_{n} A y_{n}\right)-\left(x^{*}-\lambda_{n} A x^{*}\right)\right\| \leq\left\|y_{n}-x^{*}\right\| \leq\left\|x_{n}-x^{*}\right\| .
\end{aligned}
$$

Hence we obtain that

$$
\begin{aligned}
\left\|x_{n+1}-x^{*}\right\| & =\left\|\alpha_{n}\left(f\left(W_{n} x_{n}\right)-x^{*}\right)+\beta_{n}\left(x_{n}-x^{*}\right)+\gamma_{n}\left(W_{n} u_{n}-x^{*}\right)\right\| \\
& \leq \alpha_{n}\left\|f\left(W_{n} x_{n}\right)-x^{*}\right\|+\beta_{n}\left\|x_{n}-x^{*}\right\|+\gamma_{n}\left\|W_{n} u_{n}-x^{*}\right\| \\
& \leq \alpha_{n}\left\|f\left(W_{n} x_{n}\right)-f\left(x^{*}\right)\right\|+\alpha_{n}\left\|f\left(x^{*}\right)-x^{*}\right\|+\beta_{n}\left\|x_{n}-x^{*}\right\|+\gamma_{n}\left\|u_{n}-x^{*}\right\| \\
& \leq \alpha_{n} \beta\left\|x_{n}-x^{*}\right\|+\alpha_{n}\left\|f\left(x^{*}\right)-x^{*}\right\|+\beta_{n}\left\|x_{n}-x^{*}\right\|+\gamma_{n}\left\|x_{n}-x^{*}\right\| \\
& =(1-\beta) \alpha_{n} \frac{\left\|f\left(x^{*}\right)-x^{*}\right\|}{1-\beta}+\left[1-(1-\beta) \alpha_{n}\right]\left\|x_{n}-x^{*}\right\| \\
& \leq \max \left\{\left\|x_{n}-x^{*}\right\|, \frac{\left\|f\left(x^{*}\right)-x^{*}\right\|}{1-\beta}\right\} \leq \max \left\{\left\|x_{0}-x^{*}\right\|, \frac{\left\|f\left(x^{*}\right)-x^{*}\right\|}{1-\beta}\right\} .
\end{aligned}
$$

Therefore $\left\{x_{n}\right\}$ is bounded, so are $\left\{y_{n}\right\}$ and $\left\{z_{n}\right\}$.

Step $2\left(\left\|x_{n+1}-x_{n}\right\| \rightarrow 0\right)$. Setting $x_{n+1}=\beta_{n} x_{n}+\left(1-\beta_{n}\right) V_{n}$ for all $n \geq 0$. It follows that

$$
\begin{aligned}
V_{n+1}-V_{n}= & \frac{x_{n+2}-\beta_{n+1} x_{n+1}}{1-\beta_{n+1}}-\frac{x_{n+1}-\beta_{n} x_{n}}{1-\beta_{n}} \\
= & \frac{\alpha_{n+1} f\left(W_{n+1} x_{n+1}\right)+\gamma_{n+1} W_{n+1} u_{n+1}}{1-\beta_{n+1}}-\frac{\alpha_{n} f\left(W_{n} x_{n}\right)+\gamma_{n} W_{n} u_{n}}{1-\beta_{n}} \\
= & \frac{\alpha_{n+1} f\left(W_{n+1} x_{n+1}\right)}{1-\beta_{n+1}}-\frac{\alpha_{n} f\left(W_{n} x_{n}\right)}{1-\beta_{n}}+\frac{\gamma_{n+1}}{1-\beta_{n+1}}\left(W_{n+1} u_{n+1}-W_{n+1} u_{n}\right) \\
& +\left(\frac{\gamma_{n+1}}{1-\beta_{n+1}}-\frac{\gamma_{n}}{1-\beta_{n}}\right) W_{n+1} u_{n}+\frac{\gamma_{n}}{1-\beta_{n}}\left(W_{n+1} u_{n}-W_{n} u_{n}\right),
\end{aligned}
$$


which implies that

$$
\begin{aligned}
\left\|V_{n+1}-V_{n}\right\| \leq & \frac{\alpha_{n+1}}{1-\beta_{n+1}}\left(\left\|f\left(W_{n+1} x_{n+1}\right)\right\|+\left\|W_{n+1} u_{n}\right\|\right) \\
& +\frac{\alpha_{n}}{1-\beta_{n}}\left(\left\|f\left(W_{n} x_{n}\right)\right\|+\left\|W_{n+1} u_{n}\right\|\right) \\
& +\frac{\gamma_{n+1}}{1-\beta_{n+1}}\left\|u_{n+1}-u_{n}\right\|+\frac{\gamma_{n}}{1-\beta_{n}}\left\|W_{n+1} u_{n}-W_{n} u_{n}\right\| .
\end{aligned}
$$

Now we estimate $\left\|u_{n+1}-u_{n}\right\|$ and $\left\|W_{n+1} u_{n}-W_{n} u_{n}\right\|$.

From (3.1), since $T_{i}$ and $U_{n, i}$ are nonexpansive, we have

$$
\begin{aligned}
\left\|W_{n+1} u_{n}-W_{n} u_{n}\right\| & =\left\|\xi_{1} T_{1} U_{n+1,2} u_{n}-\xi_{1} T_{1} U_{n, 2} u_{n}\right\| \\
& \leq \xi_{1}\left\|U_{n+1,2} u_{n}-U_{n, 2} u_{n}\right\| \\
& =\xi_{1}\left\|\xi_{2} T_{2} U_{n+1,3} u_{n}-\xi_{2} T_{2} U_{n, 3} u_{n}\right\| \\
& \leq \xi_{1} \xi_{2}\left\|U_{n+1,3} u_{n}-U_{n, 3} u_{n}\right\| \\
& \leq \cdots \\
& \leq \xi_{1} \xi_{2} \cdots \xi_{n}\left\|U_{n+1, n+1} u_{n}-U_{n, n+1} u_{n}\right\| \\
& \leq M \prod_{i=1}^{n} \xi_{i},
\end{aligned}
$$

where $M$ is a constant such that $\sup \left\{\left\|U_{n+1, n+1} u_{n}-U_{n, n+1} u_{n}\right\|, n \geq 0\right\} \leq M$.

At the same time, we observe that

$$
\begin{aligned}
\left\|y_{n+1}-y_{n}\right\| & =\left\|P_{C}\left(z_{n+1}-\lambda_{n+1} A z_{n+1}\right)-P_{C}\left(z_{n}-\lambda_{n} A z_{n}\right)\right\| \\
& \leq\left\|\left(z_{n+1}-\lambda_{n+1} A z_{n+1}\right)-\left(z_{n}-\lambda_{n} A z_{n}\right)\right\| \\
& =\left\|\left(z_{n+1}-\lambda_{n+1} A z_{n+1}\right)-\left(z_{n}-\lambda_{n+1} A z_{n}\right)+\left(\lambda_{n}-\lambda_{n+1}\right) A z_{n}\right\| \\
& \leq\left\|\left(z_{n+1}-\lambda_{n+1} A z_{n+1}\right)-\left(z_{n}-\lambda_{n+1} A z_{n}\right)\right\|+\left|\lambda_{n}-\lambda_{n+1}\right|\left\|A z_{n}\right\| \\
& \leq\left\|z_{n+1}-z_{n}\right\|+\left|\lambda_{n}-\lambda_{n+1}\right|\left\|A z_{n}\right\|, \\
\left\|u_{n+1}-u_{n}\right\| & =\left\|P_{C}\left(y_{n+1}-\lambda_{n+1} A y_{n+1}\right)-P_{C}\left(y_{n}-\lambda_{n} A y_{n}\right)\right\| \\
& \leq\left\|\left(y_{n+1}-\lambda_{n+1} A y_{n+1}\right)-\left(y_{n}-\lambda_{n} A y_{n}\right)\right\| \\
& =\left\|\left(y_{n+1}-\lambda_{n+1} A y_{n+1}\right)-\left(y_{n}-\lambda_{n+1} A y_{n}\right)+\left(\lambda_{n}-\lambda_{n+1}\right) A y_{n}\right\| \\
& \leq\left\|y_{n+1}-y_{n}\right\|+\left|\lambda_{n}-\lambda_{n+1}\right|\left\|A y_{n}\right\| \\
& \leq\left\|z_{n+1}-z_{n}\right\|+\left|\lambda_{n}-\lambda_{n+1}\right|\left(\left\|A y_{n}\right\|+\left\|A z_{n}\right\|\right) .
\end{aligned}
$$


Since $z_{n}=S_{r} x_{n}$ and $z_{n+1}=S_{r} x_{n+1}$, from the nonexpansivity of $S_{r}$, we get

$$
\left\|z_{n+1}-z_{n}\right\| \leq\left\|x_{n+1}-x_{n}\right\| \text {. }
$$

Substituting (3.11)-(3.13) into (3.10), we have

$$
\begin{aligned}
\left\|V_{n+1}-V_{n}\right\|-\left\|x_{n+1}-x_{n}\right\| \leq & \frac{\alpha_{n+1}}{1-\beta_{n+1}}\left(\left\|f\left(W_{n+1} x_{n+1}\right)\right\|+\left\|W_{n+1} u_{n}\right\|\right) \\
& +\frac{\alpha_{n}}{1-\beta_{n}}\left(\left\|f\left(W_{n} x_{n}\right)\right\|+\left\|W_{n+1} u_{n}\right\|\right)+M \prod_{i=1}^{n} \xi_{i} \\
& +\left|\lambda_{n}-\lambda_{n+1}\right|\left(\left\|A y_{n}\right\|+\left\|A z_{n}\right\|\right) .
\end{aligned}
$$

Since $\alpha_{n} \rightarrow 0, \lambda_{n+1}-\lambda_{n} \rightarrow 0$, and $\xi_{i} \in[a, b]$, we have

$$
\limsup _{n \rightarrow \infty}\left(\left\|V_{n+1}-V_{n}\right\|-\left\|x_{n+1}-x_{n}\right\|\right) \leq 0 .
$$

Hence by Lemma 2.2, we have

$$
\lim _{n \rightarrow \infty}\left\|V_{n}-x_{n}\right\|=0
$$

Consequently,

$$
\lim _{n \rightarrow \infty}\left\|x_{n+1}-x_{n}\right\|=0 \text {. }
$$

Step $3\left(\left\|u_{n}-W u_{n}\right\| \rightarrow 0\right)$. Note that $x_{n+1}-x_{n}=\alpha_{n}\left(f\left(W_{n} x_{n}\right)-x_{n}\right)+\gamma_{n}\left(W_{n} u_{n}-x_{n}\right)$. Then we have

$$
\left\|x_{n}-W_{n} u_{n}\right\| \leq \frac{1}{\gamma_{n}}\left\{\left\|x_{n}-x_{n+1}\right\|+\alpha_{n}\left\|f\left(W_{n} x_{n}\right)-x_{n}\right\|\right\} \longrightarrow 0 .
$$

For $x^{*} \in \Gamma$, noting that $S_{r}$ is firmly nonexpansive, we have

$$
\begin{aligned}
\left\|z_{n}-x^{*}\right\|^{2} & =\left\|S_{r} x_{n}-S_{r} x^{*}\right\|^{2} \\
& \leq\left\langle S_{r} x_{n}-S_{r} x^{*}, x_{n}-x^{*}\right\rangle \\
& =\left\langle z_{n}-x^{*}, x_{n}-x^{*}\right\rangle \\
& =\frac{1}{2}\left(\left\|z_{n}-x^{*}\right\|^{2}+\left\|x_{n}-x^{*}\right\|^{2}-\left\|x_{n}-z_{n}\right\|^{2}\right),
\end{aligned}
$$

and hence

$$
\left\|z_{n}-x^{*}\right\|^{2} \leq\left\|x_{n}-x^{*}\right\|^{2}-\left\|x_{n}-z_{n}\right\|^{2}
$$

So, we have

$$
\begin{aligned}
\left\|x_{n+1}-x^{*}\right\|^{2} & \leq \alpha_{n}\left\|f\left(W_{n} x_{n}\right)-x^{*}\right\|^{2}+\beta_{n}\left\|x_{n}-x^{*}\right\|^{2}+\gamma_{n}\left\|W_{n} u_{n}-x^{*}\right\|^{2} \\
& \leq \alpha_{n}\left\|f\left(W_{n} x_{n}\right)-x^{*}\right\|^{2}+\beta_{n}\left\|x_{n}-x^{*}\right\|^{2}+\gamma_{n}\left\|u_{n}-x^{*}\right\|^{2} \\
& \leq \alpha_{n}\left\|f\left(W_{n} x_{n}\right)-x^{*}\right\|^{2}+\beta_{n}\left\|x_{n}-x^{*}\right\|^{2}+\gamma_{n}\left\|z_{n}-x^{*}\right\|^{2} \\
& \leq \alpha_{n}\left\|f\left(W_{n} x_{n}\right)-x^{*}\right\|^{2}+\beta_{n}\left\|x_{n}-x^{*}\right\|^{2}+\gamma_{n}\left(\left\|x_{n}-x^{*}\right\|^{2}-\left\|x_{n}-z_{n}\right\|^{2}\right) \\
& \leq \alpha_{n}\left\|f\left(W_{n} x_{n}\right)-x^{*}\right\|^{2}+\left\|x_{n}-x^{*}\right\|^{2}-\gamma_{n}\left\|x_{n}-z_{n}\right\|^{2}
\end{aligned}
$$


that is,

$$
\begin{aligned}
\left\|x_{n}-z_{n}\right\|^{2} & \leq \frac{1}{\gamma_{n}}\left\{\alpha_{n}\left\|f\left(W_{n} x_{n}\right)-x^{*}\right\|^{2}+\left\|x_{n}-x^{*}\right\|^{2}-\left\|x_{n+1}-x^{*}\right\|^{2}\right\} \\
& \leq \frac{1}{\gamma_{n}}\left\{\alpha_{n}\left\|f\left(W_{n} x_{n}\right)-x^{*}\right\|^{2}+\left\|x_{n+1}-x_{n}\right\|\left(\left\|x_{n}-x^{*}\right\|+\left\|x_{n+1}-x^{*}\right\|\right)\right\} \\
& \longrightarrow 0 .
\end{aligned}
$$

From (3.6), we obtain that

$$
\begin{aligned}
\left\|x_{n+1}-x^{*}\right\|^{2} & \leq \alpha_{n}\left\|f\left(W_{n} x_{n}\right)-x^{*}\right\|^{2}+\beta_{n}\left\|x_{n}-x^{*}\right\|^{2}+\gamma_{n}\left\|y_{n}-x^{*}\right\|^{2} \\
& \leq \alpha_{n}\left\|f\left(W_{n} x_{n}\right)-x^{*}\right\|^{2}+\beta_{n}\left\|x_{n}-x^{*}\right\|^{2}+\gamma_{n}\left[\left\|\left(z_{n}-\lambda_{n} A z_{n}\right)-\left(x^{*}-\lambda_{n} A x^{*}\right)\right\|^{2}\right] \\
& \leq \alpha_{n}\left\|f\left(W_{n} x_{n}\right)-x^{*}\right\|^{2}+\beta_{n}\left\|x_{n}-x^{*}\right\|^{2}+\gamma_{n}\left\{\left\|z_{n}-x^{*}\right\|^{2}+\lambda_{n}\left(\lambda_{n}-2 \beta\right)\left\|A z_{n}-A x^{*}\right\|^{2}\right\} \\
& \leq \alpha_{n}\left\|f\left(W_{n} x_{n}\right)-x^{*}\right\|^{2}+\left\|x_{n}-x^{*}\right\|^{2}+\gamma_{n} a(b-2 \beta)\left\|A z_{n}-A x^{*}\right\|^{2} .
\end{aligned}
$$

Then we have

$$
-\gamma_{n} a(b-2 \beta)\left\|A z_{n}-A x^{*}\right\|^{2} \leq \alpha_{n}\left\|f\left(W_{n} x_{n}\right)-x^{*}\right\|^{2}+\left\|x_{n}-x^{*}\right\|^{2}-\left\|x_{n+1}-x^{*}\right\|^{2} \longrightarrow 0,
$$

which implies that

$$
\lim _{n \rightarrow \infty}\left\|A z_{n}-A x^{*}\right\|=0
$$

We note that

$$
\begin{aligned}
\left\|y_{n}-x^{*}\right\|^{2}= & \left\|P_{C}\left(z_{n}-\lambda_{n} A z_{n}\right)-P_{C}\left(x^{*}-\lambda_{n} A x^{*}\right)\right\|^{2} \\
\leq & \left\langle\left(z_{n}-\lambda_{n} A z_{n}\right)-\left(x^{*}-\lambda_{n} A x^{*}\right), y_{n}-x^{*}\right\rangle \\
= & \frac{1}{2}\left\{\left\|\left(z_{n}-\lambda_{n} A z_{n}\right)-\left(x^{*}-\lambda_{n} A x^{*}\right)\right\|^{2}+\left\|y_{n}-x^{*}\right\|^{2}\right. \\
& \left.\quad-\left\|\left(z_{n}-\lambda_{n} A z_{n}\right)-\left(x^{*}-\lambda_{n} A x^{*}\right)-\left(y_{n}-x^{*}\right)\right\|^{2}\right\} \\
\leq & \frac{1}{2}\left\{\left\|z_{n}-x^{*}\right\|^{2}+\left\|y_{n}-x^{*}\right\|^{2}-\left\|\left(z_{n}-y_{n}\right)-\lambda_{n}\left(A z_{n}-A x^{*}\right)\right\|^{2}\right\} \\
= & \frac{1}{2}\left\{\left\|z_{n}-x^{*}\right\|^{2}+\left\|y_{n}-x^{*}\right\|^{2}-\left\|z_{n}-y_{n}\right\|^{2}+2 \lambda_{n}\left\langle A z_{n}-A x^{*}, z_{n}-y_{n}\right\rangle-\lambda_{n}^{2}\left\|A z_{n}-A x^{*}\right\|^{2}\right\} .
\end{aligned}
$$

Then we derive

$$
\begin{aligned}
\left\|y_{n}-x^{*}\right\|^{2} & \leq\left\|z_{n}-x^{*}\right\|^{2}-\left\|z_{n}-y_{n}\right\|^{2}+2 \lambda_{n}\left\langle A z_{n}-A x^{*}, z_{n}-y_{n}\right\rangle-\lambda_{n}^{2}\left\|A z_{n}-A x^{*}\right\|^{2} \\
& \leq\left\|x_{n}-x^{*}\right\|^{2}-\left\|z_{n}-y_{n}\right\|^{2}+2 \lambda_{n}\left\langle A z_{n}-A x^{*}, z_{n}-y_{n}\right\rangle .
\end{aligned}
$$


Hence

$$
\begin{aligned}
& \left\|x_{n+1}-x^{*}\right\|^{2} \\
& \quad \leq \alpha_{n}\left\|f\left(W_{n} x_{n}\right)-x^{*}\right\|^{2}+\beta_{n}\left\|x_{n}-x^{*}\right\|^{2}+\gamma_{n}\left\{\left\|x_{n}-x^{*}\right\|^{2}-\left\|z_{n}-y_{n}\right\|^{2}+2 \lambda_{n}\left\langle A z_{n}-A x^{*}, z_{n}-y_{n}\right\rangle\right\},
\end{aligned}
$$

which implies that

$$
\left\|z_{n}-y_{n}\right\| \leq \frac{1}{\gamma_{n}}\left\{\alpha_{n}\left\|f\left(W_{n} x_{n}\right)-x^{*}\right\|^{2}+\left\|x_{n}-x^{*}\right\|^{2}-\left\|x_{n+1}-x^{*}\right\|^{2}+2 \gamma_{n} \lambda_{n}\left\|A z_{n}-A x^{*}\right\|\left\|z_{n}-y_{n}\right\|\right\} \longrightarrow 0 .
$$

Since $\left\|y_{n}-u_{n}\right\|=\left\|P_{C}\left(z_{n}-\lambda_{n} A z_{n}\right)-P_{C}\left(y_{n}-\lambda_{n} A y_{n}\right)\right\| \leq\left\|z_{n}-y_{n}\right\|$, we have

$$
\begin{aligned}
\left\|W u_{n}-u_{n}\right\| & \leq\left\|W u_{n}-W_{n} u_{n}\right\|+\left\|W_{n} u_{n}-u_{n}\right\| \\
& \leq\left\|W u_{n}-W_{n} u_{n}\right\|+\left\|W_{n} u_{n}-x_{n}\right\|+\left\|x_{n}-z_{n}\right\|+\left\|z_{n}-y_{n}\right\|+\left\|y_{n}-u_{n}\right\| \\
& \leq\left\|W u_{n}-W_{n} u_{n}\right\|+\left\|W_{n} u_{n}-x_{n}\right\|+\left\|x_{n}-z_{n}\right\|+2\left\|z_{n}-y_{n}\right\| .
\end{aligned}
$$

Combining the above inequality, (3.18)-(3.29), and Remark 3.3, we have

$$
\lim _{n \rightarrow \infty}\left\|W u_{n}-u_{n}\right\|=0 .
$$

Step $4\left(\lim _{n \rightarrow \infty}\left\langle f\left(x^{*}\right)-x^{*}, x_{n}-x^{*}\right\rangle\right.$, where $\left.x^{*}=P_{\Gamma} f\left(x^{*}\right)\right)$. To show the above inequality, we can choose a subsequence $\left\{u_{n_{i}}\right\}$ of $\left\{u_{n}\right\}$ such that

$$
\lim _{j \rightarrow \infty}\left\langle f\left(x^{*}\right)-x^{*}, u_{n_{j}}-x^{*}\right\rangle=\limsup _{n \rightarrow \infty}\left\langle f\left(x^{*}\right)-x^{*}, u_{n}-x^{*}\right\rangle .
$$

Since $\left\{u_{n_{j}}\right\}$ is bounded, there exists a subsequence $\left\{u_{n_{j i}}\right\}$ of $\left\{u_{n_{j}}\right\}$ which converges weakly to w. Without loss of generality, we can assume that $u_{n_{j}} \rightarrow w$. From $\left\|W u_{n}-u_{n}\right\| \rightarrow 0$, we obtain $W u_{n_{j}} \rightarrow w$.

First, we show $w \in F(W)=\cap_{n=1}^{\infty} F\left(T_{n}\right)$. Assume that $\omega \notin F(W)$. Since $u_{n_{j}} \rightarrow w$ and $w \neq W w$, by Opial's condition, we have

$$
\begin{aligned}
\liminf _{j \rightarrow \infty}\left\|u_{n_{j}}-w\right\| & <\liminf _{j \rightarrow \infty}\left\|u_{n_{j}}-W w\right\| \\
& \leq \liminf _{j \rightarrow \infty}\left(\left\|u_{n_{j}}-W u_{n_{j}}\right\|+\left\|W u_{n_{j}}-W w\right\|\right) \\
& \leq \liminf _{j \rightarrow \infty}\left\|u_{n_{j}}-w\right\|,
\end{aligned}
$$

which is a contradiction. Hence we get $w \in F(W)$. By the same argument as that in the proof of [21, Theorem 3.1], we can prove that $w \in V I(A, C)$; and by the same argument as that in the proof of [14, Theorem 4.1], we also can prove that $w \in \Omega$. Hence $w \in \Gamma$.

Since $x^{*}=P_{\Gamma} f\left(x^{*}\right) \in \Gamma$ and $\left\|u_{n}-x_{n}\right\| \rightarrow 0$, we have

$$
\begin{aligned}
\limsup _{n \rightarrow \infty}\left\langle f\left(x^{*}\right)-x^{*}, x_{n}-x^{*}\right\rangle & =\lim _{j \rightarrow \infty}\left\langle f\left(x^{*}\right)-x^{*}, x_{n_{j}}-x^{*}\right\rangle \\
& =\lim _{j \rightarrow \infty}\left\langle f\left(x^{*}\right)-x^{*}, u_{n_{j}}-x^{*}\right\rangle=\left\langle f\left(x^{*}\right)-x^{*}, w-x^{*}\right\rangle \leq 0 .
\end{aligned}
$$


Step $5\left(x_{n} \rightarrow x^{*}\right.$, where $\left.x^{*}=P_{\Gamma} f\left(x^{*}\right)\right)$. From (3.3), we have

$$
\begin{aligned}
\left\|x_{n+1}-x^{*}\right\|^{2} \leq & \left\|\beta_{n}\left(x_{n}-x^{*}\right)+\gamma_{n}\left(W_{n} u_{n}-x^{*}\right)\right\|^{2}+2 \alpha_{n}\left\langle f\left(W_{n} x_{n}\right)-x^{*}, x_{n+1}-x^{*}\right\rangle \\
\leq & {\left[\beta_{n}\left\|x_{n}-x^{*}\right\|+\gamma_{n}\left\|u_{n}-x^{*}\right\|\right]^{2}+2 \alpha_{n}\left\langle f\left(W_{n} x_{n}\right)-f\left(x^{*}\right), x_{n+1}-x^{*}\right\rangle } \\
& +2 \alpha_{n}\left\langle f\left(x^{*}\right)-x^{*}, x_{n+1}-x^{*}\right\rangle \\
\leq & \left(1-\alpha_{n}\right)^{2}\left\|x_{n}-x^{*}\right\|^{2}+2 \alpha_{n}\left\|f\left(W_{n} x_{n}\right)-f\left(x^{*}\right)\right\|\left\|x_{n+1}-x^{*}\right\| \\
& +2 \alpha_{n}\left\langle f\left(x^{*}\right)-x^{*}, x_{n+1}-x^{*}\right\rangle \\
\leq & \left(1-\alpha_{n}\right)^{2}\left\|x_{n}-x^{*}\right\|^{2}+2 \alpha \alpha_{n}\left\|x_{n}-x^{*}\right\|\left\|x_{n+1}-x^{*}\right\|+2 \alpha_{n}\left\langle f\left(x^{*}\right)-x^{*}, x_{n+1}-x^{*}\right\rangle \\
\leq & \left(1-\alpha_{n}\right)^{2}\left\|x_{n}-x^{*}\right\|^{2}+\alpha \alpha_{n}\left(\left\|x_{n}-x^{*}\right\|^{2}+\left\|x_{n+1}-x^{*}\right\|^{2}\right)+2 \alpha_{n}\left\langle f\left(x^{*}\right)-x^{*}, x_{n+1}-x^{*}\right\rangle,
\end{aligned}
$$

that is,

$$
\left\|x_{n+1}-x^{*}\right\|^{2} \leq\left[1-\frac{2(1-\alpha)}{1-\alpha \alpha_{n}} \alpha_{n}\right]\left\|x_{n}-x^{*}\right\|^{2}+\frac{2(1-\alpha)}{1-\alpha \alpha_{n}} \alpha_{n}\left\{\frac{\alpha_{n}}{2(1-\alpha)}\left\|x_{n}-x^{*}\right\|^{2}+\frac{1}{1-\alpha}\left\langle f\left(x^{*}\right)-x^{*}, x_{n+1}-x^{*}\right\rangle\right\} .
$$

It is easy to see that $\sum_{n=0}^{\infty}\left(2(1-\alpha) /\left(1-\alpha \alpha_{n}\right)\right) \alpha_{n}=\infty$ and

$$
\limsup _{n \rightarrow \infty}\left\{\frac{\alpha_{n}}{2(1-\alpha)}\left\|x_{n}-x^{*}\right\|^{2}+\frac{1}{1-\alpha}\left\langle f\left(x^{*}\right)-x^{*}, x_{n+1}-x^{*}\right\rangle\right\} \leq 0
$$

Applying Lemma 2.3 and (3.34) to (3.36), we conclude that $x_{n} \rightarrow x^{*}$ as $n \rightarrow \infty$. This completes the proof.

Concerning $S_{r}$, we give the following remark.

Remark 3.6. For each $x_{1}, x_{2} \in C$, we denote $u_{1}=S_{r}\left(x_{1}\right)$ and $u_{2}=S_{r}\left(x_{2}\right)$. Then for all $y \in C$, we have

$$
\begin{gathered}
r\left[\Theta\left(u_{1}, y\right)+\varphi(y)-\varphi\left(u_{1}\right)\right]+\left\langle K^{\prime}\left(u_{1}\right)-K^{\prime}\left(x_{1}\right), \eta\left(y, u_{1}\right)\right\rangle \geq 0, \\
r\left[\Theta\left(u_{2}, y\right)+\varphi(y)-\varphi\left(u_{2}\right)\right]+\left\langle K^{\prime}\left(u_{2}\right)-K^{\prime}\left(x_{2}\right), \eta\left(y, u_{2}\right)\right\rangle \geq 0 .
\end{gathered}
$$

Taking $y=u_{2}$ in (3.38) and $y=u_{1}$ in (3.39), and adding up these two inequalities, we obtain

$$
\begin{aligned}
& r\left[\Theta\left(u_{1}, u_{2}\right)+\varphi\left(u_{2}\right)-\varphi\left(u_{1}\right)\right]+\left\langle K^{\prime}\left(u_{1}\right)-K^{\prime}\left(x_{1}\right), \eta\left(u_{2}, u_{1}\right)\right\rangle \\
& \quad+r\left[\Theta\left(u_{2}, u_{1}\right)+\varphi\left(u_{1}\right)-\varphi\left(u_{2}\right)\right]+\left\langle K^{\prime}\left(u_{2}\right)-K^{\prime}\left(x_{2}\right), \eta\left(u_{1}, u_{2}\right)\right\rangle \geq 0 .
\end{aligned}
$$

Note that $\eta\left(u_{1}, u_{2}\right)+\eta\left(u_{2}, u_{1}\right)=0$ and $\Theta\left(u_{1}, u_{2}\right)+\Theta\left(u_{2}, u_{1}\right) \leq 0$. Hence from (3.40), we deduce

$$
\left\langle K^{\prime}\left(x_{1}\right)-K^{\prime}\left(u_{1}\right), \eta\left(u_{1}, u_{2}\right)\right\rangle+\left\langle K^{\prime}\left(u_{2}\right)-K^{\prime}\left(x_{2}\right), \eta\left(u_{1}, u_{2}\right)\right\rangle \geq 0,
$$


which implies that

$$
\left\langle K^{\prime}\left(x_{1}\right)-K^{\prime}\left(x_{2}\right), \eta\left(u_{1}, u_{2}\right)\right\rangle \geq\left\langle K^{\prime}\left(u_{1}\right)-K^{\prime}\left(u_{2}\right), \eta\left(u_{1}, u_{2}\right)\right\rangle
$$

Since $K^{\prime}: C \rightarrow H$ is $\eta$-strongly monotone with constant $\mu>0$, then from (3.42), we conclude that

$$
\left\langle K^{\prime}\left(x_{1}\right)-K^{\prime}\left(x_{2}\right), \eta\left(u_{1}, u_{2}\right)\right\rangle \geq \mu\left\|u_{1}-u_{2}\right\|^{2}
$$

Take $K(x)=\|x\|^{2} / 2, \eta(y, x)=y-x$, and $\mu=1$. Then from (3.43), we have

$$
\left\langle x_{1}-x_{2}, u_{1}-u_{2}\right\rangle \geq\left\|u_{1}-u_{2}\right\|^{2}
$$

This indicates that $S_{r}$ is firmly nonexpansive.

Corollary 3.7. Let $C$ be a nonempty closed convex subset of a real Hilbert space $H$ and let $\varphi: C \rightarrow \mathbf{R}$ be a lower semicontinuous and convex functional. Let $\Theta: C \times C \rightarrow \mathbf{R}$ be an equilibrium bifunction satisfying conditions (H1)-(H3). Let $A: C \rightarrow H$ be a $\beta$-inverse-strongly monotone mapping such that $\operatorname{VI}(A, C) \cap \Omega \neq \varnothing$. Suppose $\left\{\alpha_{n}\right\},\left\{\beta_{n}\right\}$, and $\left\{\gamma_{n}\right\}$ are three sequences in $(0,1)$ with $\alpha_{n}+\beta_{n}+\gamma_{n}=$ $1, n \geq 0$. Assume that

(i) $\eta: C \times C \rightarrow H$ is Lipschitz continuous with constant $\lambda>0$ such that

(a) $\eta(x, y)+\eta(y, x)=0$ for all $x, y \in C$,

(b) $\eta(\cdot, \cdot)$ is affine in the first variable,

(c) for each fixed $y \in C, x \mapsto \eta(y, x)$ is sequentially continuous from the weak topology to the weak topology;

(ii) $K: C \rightarrow \mathbf{R}$ is $\eta$-strongly convex with constant $\sigma>0$ and its derivative $K^{\prime}$ is not only sequentially continuous from the weak topology to the strong topology but also Lipschitz continuous with constant $v>0$ such that $\sigma \geq \lambda v$;

(iii) for each $x \in C$, there exist a bounded subset $D_{x} \subset C$ and $z_{x} \in C$ such that, for any $y \in C \backslash D_{x}$,

$$
\Theta\left(y, z_{x}\right)+\varphi\left(z_{x}\right)-\varphi(y)+\frac{1}{r}\left\langle K^{\prime}(y)-K^{\prime}(x), \eta\left(z_{x}, y\right)\right\rangle<0
$$


(iv) $\lim _{n \rightarrow \infty} \alpha_{n}=0, \sum_{n=0}^{\infty} \alpha_{n}=\infty, 0<\liminf _{n \rightarrow \infty} \beta_{n} \leq \limsup _{n \rightarrow \infty} \beta_{n}<1, \lambda_{n} \in[a, b] \subset$ $(0,2 \beta)$, and $\lim _{n \rightarrow \infty}\left(\lambda_{n+1}-\lambda_{n}\right)=0$.

Let $f$ be a contraction of $C$ into itself and given $x_{0} \in C$ arbitrarily. Let the sequences $\left\{x_{n}\right\},\left\{y_{n}\right\}$, and $\left\{z_{n}\right\}$ be generated iteratively by

$$
\begin{gathered}
\Theta\left(z_{n}, x\right)+\varphi(x)-\varphi\left(z_{n}\right)+\frac{1}{r}\left\langle K^{\prime}\left(z_{n}\right)-K^{\prime}\left(x_{n}\right), \eta\left(x, z_{n}\right)\right\rangle \geq 0 \quad \forall x \in C, \\
y_{n}=P_{C}\left(z_{n}-\lambda_{n} A z_{n}\right), \\
x_{n+1}=\alpha_{n} f\left(x_{n}\right)+\beta_{n} x_{n}+\gamma_{n} P_{C}\left(y_{n}-\lambda_{n} A y_{n}\right) \quad \forall n \geq 0 .
\end{gathered}
$$

Then the sequence $\left\{x_{n}\right\}$ generated by (3.46) converges strongly to $x^{*}=P_{\Gamma} f\left(x^{*}\right)$, where $\Gamma=V I(A, C) \cap$ $\Omega$ provided that $S_{r}$ is firmly nonexpansive.

Proof. Take $T_{n} x=x$ for all $n=1,2, \ldots$, and for all $x \in C$ in (3.1). Then $W_{n} x=x$ for all $x \in C$. The conclusion follows immediately from Theorem 3.5. This completes the proof.

Corollary 3.8. Let $C$ be a nonempty closed convex subset of a real Hilbert space $H$. Let $T_{1}, T_{2}, \ldots$ be an infinite family of nonexpansive mappings of $C$ into itself. Let $A: C \rightarrow H$ be a $\beta$-inverse-strongly monotone mapping such that $\cap_{n=1}^{\infty} F\left(T_{n}\right) \cap V I(A, C) \neq \varnothing$. Suppose $\left\{\alpha_{n}\right\},\left\{\beta_{n}\right\}$, and $\left\{\gamma_{n}\right\}$ are three sequences in $(0,1)$ with $\alpha_{n}+\beta_{n}+\gamma_{n}=1, n \geq 0$. Assume that

(i) $\lim _{n \rightarrow \infty} \alpha_{n}=0$ and $\sum_{n=0}^{\infty} \alpha_{n}=\infty$;

(ii) $0<\liminf _{n \rightarrow \infty} \beta_{n} \leq \lim \sup _{n \rightarrow \infty} \beta_{n}<1$;

(iii) $\lambda_{n} \in[a, b] \subset(0,2 \beta)$ and $\lim _{n \rightarrow \infty}\left(\lambda_{n+1}-\lambda_{n}\right)=0$.

Let $f$ be a contraction of $C$ into itself and given $x_{0} \in C$ arbitrarily. Then the sequence $\left\{x_{n}\right\}$, generated iteratively by

$$
\begin{gathered}
y_{n}=P_{C}\left(x_{n}-\lambda_{n} A x_{n}\right), \\
x_{n+1}=\alpha_{n} f\left(W_{n} x_{n}\right)+\beta_{n} x_{n}+\gamma_{n} W_{n} P_{C}\left(y_{n}-\lambda_{n} A y_{n}\right) \quad \forall n \geq 0,
\end{gathered}
$$

converges strongly to $x^{*}=P_{\Gamma} f\left(x^{*}\right)$, where $\Gamma=\cap_{n=1}^{\infty} F\left(T_{n}\right) \cap V I(A, C)$.

Proof. Set $\varphi(x)=0$ and $\Theta(x, y)=0$ for all $x, y \in C$ and put $r=1$. Take $K(x)=\|x\|^{2} / 2$ and $\eta(y, x)=y-x$ for all $x, y \in C$. Then we have $z_{n}=P_{C} x_{n}=x_{n}$. Hence the conclusion follows. This completes the proof.

\section{Acknowledgments}

The authors are extremely grateful to the anonymous referees and Professor T. Suzuki for their useful comments and suggestions. The first author was partially supported by National Natural Science Foundation of China, Grant no. 10771050. The second author was partially supported by the Grant no. NSC 96-2221-E-230-003. 


\section{References}

[1] E. Blum and W. Oettli, "From optimization and variational inequalities to equilibrium problems," The Mathematics Student, vol. 63, no. 1-4, pp. 123-145, 1994.

[2] L.-C. Zeng, S.-Y. Wu, and J.-C. Yao, "Generalized KKM theorem with applications to generalized minimax inequalities and generalized equilibrium problems," Taiwanese Journal of Mathematics, vol. 10, no. 6, pp. 1497-1514, 2006.

[3] O. Chadli, N. C. Wong, and J.-C. Yao, "Equilibrium problems with applications to eigenvalue problems," Journal of Optimization Theory and Applications, vol. 117, no. 2, pp. 245-266, 2003.

[4] O. Chadli, S. Schaible, and J.-C. Yao, "Regularized equilibrium problems with application to noncoercive hemivariational inequalities," Journal of Optimization Theory and Applications, vol. 121, no. 3, pp. 571-596, 2004.

[5] I. V. Konnov, S. Schaible, and J.-C. Yao, "Combined relaxation method for mixed equilibrium problems," Journal of Optimization Theory and Applications, vol. 126, no. 2, pp. 309-322, 2005.

[6] P. L. Combettes and S. A. Hirstoaga, "Equilibrium programming in Hilbert spaces," Journal of Nonlinear and Convex Analysis, vol. 6, no. 1, pp. 117-136, 2005.

[7] S. D. Flåm and A. S. Antipin, "Equilibrium programming using proximal-like algorithms," Mathematical Programming, vol. 78, no. 1, pp. 29-41, 1996.

[8] S. Takahashi and W. Takahashi, "Viscosity approximation methods for equilibrium problems and fixed point problems in Hilbert spaces," Journal of Mathematical Analysis and Applications, vol. 331, no. 1, pp. 506-515, 2007.

[9] O. Chadli, I. V. Konnov, and J.-C. Yao, "Descent methods for equilibrium problems in a Banach space," Computers \& Mathematics with Applications, vol. 48, no. 3-4, pp. 609-616, 2004.

[10] X. P. Ding, Y. C. Lin, and J.-C. Yao, "Predictor-corrector algorithms for solving generalized mixed implicit quasi-equilibrium problems," Applied Mathematics and Mechanics, vol. 27, no. 9, pp. 1157-1164, 2006.

[11] A. Tada and W. Takahashi, "Strong convergence theorem for an equilibrium problem and a nonexpansive mapping," in Nonlinear Analysis and Convex Analysis, W. Takahashi and T. Tanaka, Eds., pp. 609-617, Yokohama Publishers, Yokohama, Japan, 2007.

[12] Y. Yao, Y.-C. Liou, and J.-C. Yao, "Convergence theorem for equilibrium problems and fixed point problems of infinite family of nonexpansive mappings," Fixed Point Theory and Applications, vol. 2007, Article ID 64363, 12 pages, 2007.

[13] P. L. Combettes and A. S. Hirstoaga, "Equilibrium programming using proximal-like algorithms," Mathematical Programming, vol. 78, no. 1, pp. 29-41, 1997.

[14] L.-C. Zeng and J.-C. Yao, "A hybrid iterative scheme for mixed equilibrium problems and fixed point problems," Journal of Computational and Applied Mathematics, vol. 214, no. 1, pp. 186-201, 2008.

[15] L.-C. Zeng and J.-C. Yao, "Strong convergence theorem by an extragradient method for fixed point problems and variational inequality problems," Taiwanese Journal of Mathematics, vol. 10, no. 5, pp. 1293-1303, 2006.

[16] Y. Yao and J.-C. Yao, "On modified iterative method for nonexpansive mappings and monotone mappings," Applied Mathematics and Computation, vol. 186, no. 2, pp. 1551-1558, 2007.

[17] T. Suzuki, "Strong convergence theorems for infinite families of nonexpansive mappings in general Banach spaces," Fixed Point Theory and Applications, vol. 2005, no. 1, pp. 103-123, 2005.

[18] H.-K. Xu, "Viscosity approximation methods for nonexpansive mappings," Journal of Mathematical Analysis and Applications, vol. 298, no. 1, pp. 279-291, 2004.

[19] P. K. F. Kuhfittig, "Common fixed points of nonexpansive mappings by iteration," Pacific Journal of Mathematics, vol. 97, no. 1, pp. 137-139, 1981.

[20] K. Shimoji and W. Takahashi, "Strong convergence to common fixed points of infinite nonexpansive mappings and applications," Taiwanese Journal of Mathematics, vol. 5, no. 2, pp. 387-404, 2001.

[21] N. Nadezhkina and W. Takahashi, "Weak convergence theorem by an extragradient method for nonexpansive mappings and monotone mappings," Journal of Optimization Theory and Applications, vol. 128, no. 1, pp. 191-201, 2006. 